\title{
STABILIZATION CRITERIA FOR CONTINUOUS LINEAR TIME-INVARIANT SYSTEMS WITH CONSTANT LAGS
}

\author{
M. DE LA SEN
}

Received 1 July 2005; Accepted 10 October 2005

Some criteria for asymptotic stability of linear and time-invariant systems with constant point delays are derived. Such criteria are concerned with the properties of robust stability related to two relevant auxiliary delay-free systems which are built by deleting the delayed dynamics or considering that the delay is zero. Explicit asymptotic stability results, easy to test, are given for both the unforced and closed-loop systems when the stabilizing controller for one of the auxiliary delay-free systems is used for the current time-delay system. The proposed techniques include frequency domain analysis techniques including the use of $H_{\infty}$ norms.

Copyright (c) 2006 M. De La Sen. This is an open access article distributed under the Creative Commons Attribution License, which permits unrestricted use, distribution, and reproduction in any medium, provided the original work is properly cited.

\section{Introduction}

Time-delay models frequently appear in problems like transportation or population growth. Also, circuits which include elements with delays have become relevant due to the increase in performance of VLSI systems. Two typical types of circuits with delays are transmission lines and partial element equivalent circuits. Stability criteria have been proposed for such systems from Lyapunov's theory or from algebraic formulations. It is well-known that the stability of linear and time-delay systems is difficult to test because it is associated with transcendental characteristic equations which possess, in general, infinitely many characteristic roots; and thus time-delay systems are, in general, infinitedimensional (see, for instance, $[1-8,12,13,15,18,21,22]$ ). Therefore, many of the existing stability tests are difficult to apply in practice.

In that context, several approaches for sufficiency-type stability criteria have been established in the literature (see, for instance, $[1-3,12,13,15,18,21])$. In general, those criteria include some free tuning scalar and/or matrix parameters and there is a lot of work concerned with providing as less conservative stability conditions as possible while reducing simultaneously the number of tuning parameters (see, for instance, [22] and references therein). In that paper, stability criteria for linear time-invariant systems with 
multiple delays are established. One of the main results is for stability independence of delay, namely, all the characteristic roots are guaranteed to lie in $\operatorname{Re} s<0$ for all finite delays $(k=1,2, \ldots, \sigma)$. The second main result consists of sufficiency-type conditions for asymptotic stability dependent on the delays. All the results are derived in the frequency domain based on the use of Rouchés theorem for the zeros of a complex variable function related to another one which is taken as reference on some appropriate domain, $[10,11,16,17,19,20]$. In this context, the number of zeros of the characteristic quasipolynomial is compared to that of the characteristic polynomials of an auxiliary delay-free system on the closed right half-plane. If both numbers coincide, then the current system with delays is globally asymptotically stable provided that the auxiliary delay-free system is exponentially stable and the delayed dynamics size is sufficiently small characterized in terms of norms. This auxiliary system plays the role of a nominal system and the delayed dynamics is considered as a disturbance of those nominal systems in a robustness stability context. Two auxiliary delay-free systems having physical interpretations are stated as potential nominal systems for robustness analysis. One of those systems is obtained by neglecting the overall delayed dynamics, while the other one is obtained from the current system for zero delay. The obtained results are extended to the asymptotic stabilization of the current delay system by a nominal controller which asymptotically stabilizes at least one of the above-mentioned delay-free systems. If the considered auxiliary delay-free system is exponentially stable and the contribution of the unmodeled dynamics to the characteristic equation of the delay system is sufficiently small for all frequencies compared to that of the auxiliary delay-free system, then such a system remains asymptotically stable. The basic robust asymptotic stability results are given and commented in Section 3, while proved in the appendix. Some illustrative examples are given in Section 4 and, finally, conclusions end the paper.

\section{Problem statement}

Consider the linear and time-invariant system with $\sigma$ commensurate delays $h_{i}=i h ; i=$ $1,2, \ldots, \sigma$, with state-space description:

$$
\dot{x}(t)=\mathbf{A}_{0} x(t)+\rho \sum_{i=1}^{\sigma} \mathbf{A}_{\mathbf{i}} x(t-i h)+b u(t), \quad y(t)=c^{T} x(t)
$$

for basic delay $h \geq 0$, with initial condition $\varphi:[-\sigma h, 0] \rightarrow \mathbb{R}^{n}$ where $\varphi$ is a real vector piecewise continuous function possibly possessing bounded discontinuities on a subset of measure zero of $[-\sigma h, 0] \rightarrow \mathbb{R}, x(t) \in \mathbb{R}^{n}, u(t) \in \mathbb{R}$, and $y(t) \in \mathbb{R}$ are the $n$-state vector and scalar input and output at time $t$, respectively, and $b \in \mathbb{R}^{n}, c \in \mathbb{R}^{n}$, and $\mathbf{A}_{\mathbf{i}} \in \mathbb{R}^{n x n}$ $(i=1,2, \ldots, \sigma)$ are matrices of constant real entries. The scalar parameter $\rho$ quantifies the amount of delayed dynamics for given not all zero matrices $\mathbf{A}_{\mathbf{i}}(i=1,2, \ldots, \sigma)$. For $\rho=0$ and $h=0$, the delayed system (2.1) becomes the auxiliary delay-free systems

$$
\begin{gathered}
\dot{x}(t)=\mathbf{A}_{0} x(t)+b u(t) ; \quad y(t)=c^{T} x(t), \\
\dot{x}(t)=\left(\mathbf{A}_{0}+\rho \sum_{i=1}^{\sigma} \mathbf{A}_{\mathbf{i}}\right) x(t)+b u(t) ; \quad y(t)=c^{T} x(t),
\end{gathered}
$$


respectively. For $\rho=0$, the dynamic system (2.3) reduces to (2.2). The main objective of the subsequent study is to relate the asymptotic stability properties of the system (2.1) with those of the delay-free system (2.2) provided that it is exponentially stable. The study is then extended to investigate conditions which ensure that the class of (potentially memoryless, namely, without delayed dynamics) linear controllers that stabilizes the delay-free system (2.2) also stabilizes (2.1) by stating the problem as a robust stability problem. For this purpose, the delayed dynamics of (2.1) is considered as a perturbation of that of the nominal delay-free system (2.2). Parallel robust stability results are obtained by comparing the system (2.1) to the auxiliary system (2.3). It is proved that any controller which stabilizes (2.2) exponentially, it also stabilizes asymptotically (2.1) for all $\rho \in\left[-\rho_{0}^{*}, \rho_{0}^{*}\right]$ and some real $\rho_{0}^{*}>0$. The following result, which is proved in the appendix, is related to the input-output description of the system (2.1) compared to the transfer function of the system (2.2).

Lemma 2.1. The following two items hold.

(i) The transfer function of (2.1) is given by

$$
P(s)=\frac{B\left(s, e^{-h s}\right)}{A\left(s, e^{-h s}\right)}=\frac{P_{0}(s)+\rho \Delta_{B}\left(s, e^{-h s}\right)}{1+\rho \Delta_{A}\left(s, e^{-h s}\right)},
$$

where $P_{0}(s)=N(s) / D(s)=c^{T}\left(s I-\mathbf{A}_{0}\right)^{-1} b$ is the transfer function of the auxiliary delay-free system (2.2) and

$$
\begin{aligned}
& \Delta_{B}\left(s, e^{-h s}\right)=\frac{B^{\prime}\left(s, e^{-h s}\right)}{D(s)}=\frac{1}{D(s)}\left(\sum_{i=1}^{q} B_{i}^{*^{\prime}}(s) e^{-i h s}\right)=\frac{1}{D(s)}\left(\sum_{k=0}^{m} B_{k}^{\prime}\left(e^{-h s}\right) s^{k}\right), \\
& \Delta_{A}\left(s, e^{-h s}\right)=\frac{A^{\prime}\left(s, e^{-h s}\right)}{D(s)}=\frac{1}{D(s)}\left(\sum_{i=1}^{q} A_{i}^{*^{\prime}}(s) e^{-i h s}\right)=\frac{1}{D(s)}\left(\sum_{k=0}^{n} A_{k}^{\prime}\left(e^{-h s}\right) s^{k}\right),
\end{aligned}
$$

with $B_{i}^{*^{\prime}}(s)=\sum_{k=0}^{m} b_{k i}^{\prime} s^{k}, B_{\ell}^{\prime}\left(e^{-h s}\right)=\sum_{k=1}^{q} b_{\ell k}^{\prime} e^{-k h s}$ for $i=1,2, \ldots, q(\leq n \sigma) ; l=0,1, \ldots, m$; $A_{i}^{*^{\prime}}(s)=\sum_{k=0}^{n} a_{k i}^{\prime} s^{k}, A_{\ell}^{\prime}\left(e^{-h s}\right)=\sum_{k=1}^{q} a_{\ell k}^{\prime} e^{-k h s}$ for $i=1,2, \ldots, q ; l=0,1, \ldots, n$; and $D(s)=$ $A_{0}^{*}(s)=\operatorname{det}\left(s I-\mathbf{A}_{0}\right)=\sum_{i=0}^{n} a_{i 0} s^{i}$ is a monic polynomial; that is, $a_{n 0}=1$, the real coefficients $a_{(\cdot)}^{\prime}$ and $b_{(\cdot)}^{\prime}$ being dependent on powers of $\rho$ but converging to real limit values being independent of $\rho$ as $\rho \rightarrow 0$.

(ii) The transfer function of (2.1) is equivalently given by

$$
P(s)=\frac{B\left(s, e^{-h s}\right)}{A\left(s, e^{-h s}\right)}=\frac{\bar{P}_{0}(s)+\rho \bar{\Delta}_{B}\left(s, e^{-h s}\right)}{1+\rho \bar{\Delta}_{A}\left(s, e^{-h s}\right)},
$$

where $\bar{P}_{0}(s)=\bar{N}(s) / \bar{D}(s)=c^{T}\left(s I-\mathbf{A}_{0}-\rho \sum_{i=1}^{q} \mathbf{A}_{\mathbf{i}}\right)^{-1} b$ is the transfer function of the auxiliary delay-free system (2.3) with denominator polynomial $\bar{D}(s)=\operatorname{det}\left(s I-\mathbf{A}_{\mathbf{0}}-\rho \sum_{i=1}^{q} \mathbf{A}_{\mathbf{i}}\right)$; 
and

$$
\begin{aligned}
& \bar{\Delta}_{B}\left(s, e^{-h s}\right)=\frac{1}{\bar{D}(s)}\left(\sum_{i=1}^{q} B_{i}^{*^{\prime}}(s)\left(e^{-i h s}-1\right)\right)=\frac{1}{\bar{D}(s)}\left(\sum_{k=0}^{m} B_{k}^{\prime}\left(e^{-h s}\right)\left(s^{k}-1\right)\right), \\
& \bar{\Delta}_{A}\left(s, e^{-h s}\right)=\frac{1}{\bar{D}(s)}\left(\sum_{i=1}^{q} A_{i}^{*^{\prime}}(s)\left(e^{-i h s}-1\right)\right)=\frac{1}{\bar{D}(s)}\left(\sum_{k=0}^{n} A_{k}^{\prime}\left(e^{-h s}\right)\left(s^{k}-1\right)\right) .
\end{aligned}
$$

Note from (2.5) into (2.4) that the numerator and denominator quasipolynomials $B\left(s, e^{-h s}\right)$ and $A\left(s, e^{-h s}\right)$ of the transfer function of $(2.1)$, that is, $P(s)$, may be expanded into powers of $s$, with polynomial coefficients in $e^{-h s}$, or equivalently, into powers of $e^{-h s}$ with polynomial coefficients in $s$. On the other hand, note that there are two equivalent alternative expressions for $P(s)$ related to the transfer functions of the auxiliary delay-free systems (2.2) or (2.3), $P_{0}(s)$ ( $\rho=0$ in (2.1), i.e., the system is free of delayed dynamics) and $\bar{P}_{0}(s)(h=0$ in $(2.1)$, i.e., the system operates with zero delay), respectively. Those two characterizations allow the formulations of two alternative sets of robust asymptotic stability conditions for the unforced and forced (2.1) with respect to the delay-free systems (2.2) and (2.3).

\section{Robust stability results}

In this section, some results concerned with robust stability for the unforced system (2.1) are given provided that one of the delay-free systems (2.2) or (2.3) is exponentially stable. Some obtained robust stability results are of independent of delay-type while others are formulated as dependent on the delay ones. The results may be tested in practice with simple calculations and they are also extended for the closed-loop system obtained from (2.1) with a linear (nominal) controller which stabilizes either (2.2) or (2.3). In order to establish and prove the subsequent result, define real constants $m_{A}, m_{A}^{\prime}, m_{B}$, and $m_{B}^{\prime}$ as follows:

$$
\begin{aligned}
& m_{A}:=\operatorname{Max}_{1 \leq i \leq q} \operatorname{Sup}_{\omega \in \mathbb{R}_{0}^{+}}\left(\left|\frac{A_{i}^{*^{\prime \prime}}(j \omega)}{D(j \omega)}\right|\right) ; \quad m_{B}:=\operatorname{Max}_{1 \leq i \leq q} \operatorname{Sup}_{\omega \in \mathbb{R}_{0}^{+}}\left(\left|\frac{B_{i}^{*^{\prime \prime}}(j \omega)}{N(j \omega)}\right|\right) ; \\
& m_{A}^{\prime}= \begin{cases}q \frac{1-\rho_{0}^{q+1}}{1-\rho_{0}} \operatorname{Max}_{1 \leq i \leq q} \operatorname{Sup}_{\omega \in \mathbb{R}_{0}^{+}}\left(\left|\frac{\bar{A}_{i}^{*^{\prime \prime}}(j \omega)}{D(j \omega)}\right|\right) & \text { if } \rho_{0}<1, \\
q\left(\sum_{k=0}^{q} \rho_{0}^{k}\right) \operatorname{Max}_{1 \leq i \leq q} \operatorname{Sup}_{\omega \in \mathbb{R}_{0}^{+}}\left(\left|\frac{\bar{A}_{i}^{*^{\prime \prime}}(j \omega)}{D(j \omega)}\right|\right) & \text { if } \rho_{0} \geq 1 ;\end{cases} \\
& m_{B}^{\prime}= \begin{cases}q \frac{1-\rho_{0}^{q+1}}{1-\rho_{0}} \operatorname{Max}_{1 \leq i \leq q} \operatorname{Sup}_{\omega \in \mathbb{R}_{0}^{+}}\left(\left|\frac{\bar{B}_{i}^{*^{\prime \prime}}(j \omega)}{N(j \omega)}\right|\right) & \text { if } \rho_{0}<1, \\
q\left(\sum_{k=0}^{q} \rho_{0}^{k}\right) \operatorname{Max}_{1 \leq i \leq q} \operatorname{Sup}_{\omega \in \mathbb{R}_{0}^{+}}\left(\left|\frac{\bar{B}_{i}^{*^{\prime \prime}}(j \omega)}{N(j \omega)}\right|\right) & \text { if } \rho_{0} \geq 1 ;\end{cases}
\end{aligned}
$$


with $N(s)=B_{0}^{*}(s)$ and $D(s)=A_{0}^{*}(s)$ being the numerator and denominator polynomials of $P_{0}(s)$ for the system (2.2). Note by direct inspection of (3.1) that the above constants are monotonically nondecreasing for all real $\rho_{0} \in[0,1) \cup[1, \infty)$ and all positive integer $q$. The basic robust stability result compares the global asymptotic stability of (2.1) to the exponential one of (2.2) and it is established as follows.

Theorem 3.1 (global asymptotic robust stability independence of delay from the stability of the delay-free system (2.2)). The following items hold.

(i) Assume that the unforced (i.e., $u \equiv 0$ ) delay-free system (2.2) is stable with no poles on the imaginary axis (i.e., globally exponentially stable) and with $H_{\infty}$-norm $\gamma_{0}:=\left\|P_{0}\right\|_{\infty}=$ $\operatorname{Max}_{\omega \in \mathbb{R}_{0}^{+}}\left(\left|P_{0}(j \omega)\right|\right)<\infty$ with $\mathbb{R}_{0}^{+}$being the set of nonnegative real numbers. Thus, the delayed system is globally asymptotically stable independent of delay; that is, for all $h \in[0, \infty)$, if $\rho \in\left[-\rho_{0}^{*}, \rho_{0}^{*}\right]$ with $\rho_{0}^{*}=\operatorname{Min}\left(\rho_{0}, 1 / \rho_{0}^{\prime}\right)>0, \rho_{0}>0$ being a design parameter, and $\rho_{0}^{\prime}\left(\rho_{0}\right)$ being dependent on $\rho_{0}$ from (3.1) defined by

$$
\rho_{0}^{\prime}=\gamma_{0}^{-1}\left(m_{A}+m_{A}^{\prime}\right)
$$

(ii) Assume that the pair $\left(\mathbf{A}_{\mathbf{0}}, b\right)$ is stabilizable with $D(s)=A_{0}^{*}(s)$ with no zeros on the imaginary axis and that $c(s)$ is the transfer function of a linear stabilizing feed-forward controller for the transfer function $P_{0}(s)$ of (2.2) to compose the closed-loop transfer function $T_{0}(s)=P_{0}(s) c(s) /\left(1+P_{0}(s) c(s)\right)$ of $H_{\infty}$-norm $\gamma:=\left\|T_{0}\right\|_{\infty}$. Assume also that $P_{0}(s)$ and $P(s)$ have both the same number $0 \leq n_{u} \leq n$ of unstable poles. Thus, the closed-loop delayed system of transfer function $T(s)=P(s) c(s) /(1+P(s) c(s))$, obtained from (2.1) with the same controller, remains globally asymptotically stable independent of delay for all $\rho \in\left[-\rho_{0}^{*}, \rho_{0}^{*}\right]$ with $\rho_{0}^{*}=\operatorname{Min}\left(\rho_{0}, 1 / \rho_{0 T}^{\prime}\right), \rho_{0}$ being a design parameter, and $\rho_{0}^{\prime}$ being redefined as

$$
\rho_{0 T}^{\prime}= \begin{cases}(1-\gamma)\left(m_{A T}+m_{A T}^{\prime}\right)+\gamma\left(m_{B T}+m_{B T}^{\prime}\right) & \text { if } \gamma<1, \\ (1+\gamma)\left(m_{A T}+m_{A T}^{\prime}\right)+\gamma\left(m_{B T}+m_{B T}^{\prime}\right) & \text { if } \gamma \geq 1 .\end{cases}
$$

The proof is given in the appendix.

Note that typically, the nominal stabilizing controller of transfer function is delayfree which implies that it is memoryless, but nothing about is specifically assumed in the above result. Note also that the numerator and denominator quasipolynomials of the open-loop and closed-loop transfer functions of (2.1) may be calculated equivalently as polynomials in $s$ of polynomial coefficients in $e^{-h s}$, or vice versa. Concerned with a judicious practical application of Theorem 3.1, note the following from (3.1), (3.2). If $\rho_{0} \in[0,1)$ and $q \geq 2$, then if the value of the design parameter $\rho_{0}$ becomes increased (decreased), then that of $\rho_{0}^{\prime}$ decreases 8 (increases) so of $\rho_{0}^{\prime-1}$ increases (decreases). Thus, a practical test from Theorem 3.1 may reduce to choosing $\rho_{0}=1$ and then $\rho_{0}^{*}=1 / \rho_{0}^{\prime}$. This strategy works since from the above discussion, a decrease of the value of $\rho_{0}$ corresponds with a decrease in that of $\rho_{0}^{\prime-1}$ so that an improved test is not required to increase the value of a potential $\rho_{0}^{*}$. If $\rho_{0} \geq 1$ then an increase of value in $\rho_{0}$ implies a decrease in that of $\rho_{0}^{\prime-1}$ so that it suffices a test for $\rho_{0}^{\prime}$ with $\rho_{0}=1$. For the design of a feedback system with a linear potentially memoryless controller (Theorem 3.1(ii)), which also stabilizes (2.2), the same above conclusion remains valid for stability testing. Note also that Theorem 3.1(ii) may 
be applied either for an unforced exponentially stable (2.2) or for an unstable one with no critically stable poles. In this second case, the number of unstable poles of the unforced system (2.2) has to be identical to the number of unstable poles of the unforced system (2.1) which has then to be finite as assumed in the theorem.

Thus, the subsequent alternative stability result to Theorem 3.1, but formulated as a dependent on the delays result, is established as follows.

Theorem 3.2 (global asymptotic stability dependent on the delays). The stability results of items (i), (ii) of Theorem 3.1 also hold, particularized for a basic delay $h$ if the constants $m_{A}, m_{A}^{\prime}$ are redefined dependent on delay with the replacements $q \rightarrow n, A_{i}^{* \prime \prime}(j \omega) \rightarrow$ $A_{i}^{\prime \prime}\left(e^{-j \omega h}\right) \omega^{i}, \bar{A}_{i}^{* \prime \prime}(j \omega) \rightarrow \bar{A}_{i}^{\prime \prime}\left(e^{-j \omega h}\right) \omega^{i}$; and $m_{B}$ and $m_{B}^{\prime}$ of (3.1) are redefined with the replacements $q \rightarrow m, B_{i}^{*^{\prime \prime}}(j \omega) \rightarrow B_{i}^{\prime \prime}\left(e^{-j \omega h}\right) \omega^{i}, \bar{B}_{i}^{*^{\prime \prime}}(j \omega) \rightarrow \bar{B}_{i}^{\prime \prime}\left(e^{-j \omega h}\right) \omega^{i}$.

The proof of Theorem 3.2 is close to that of Theorem 3.1 and sketched in the appendix. Note that the numerator and denominator polynomials of (2.1) of real coefficients are of the forms

$$
\begin{aligned}
& B\left(s, e^{-h s}\right)=\sum_{i=0}^{m} \sum_{k=0}^{q} b_{i k} e^{-k h s} s^{i}=\sum_{i=0}^{n} b_{i 0} s^{i}+\rho \sum_{i=1}^{m} \sum_{k=0}^{q} b_{i k}^{\prime}(\rho) e^{-k h s} s^{i}, \\
& A\left(s, e^{-h s}\right)=\sum_{i=0}^{n} \sum_{k=0}^{q} a_{i k} e^{-k h s} s^{i}=\sum_{i=0}^{n} a_{i 0} s^{i}+\rho \sum_{i=1}^{n} \sum_{k=0}^{q} a_{i k}^{\prime}(\rho) e^{-k h s} s^{i},
\end{aligned}
$$

where the following decompositions hold:

$$
a_{i k}^{\prime}(\rho)=a_{i k}^{\prime}(0)+a_{i k}^{\prime \prime}(\rho) ; \quad b_{i k}^{\prime}(\rho)=b_{i k}^{\prime}(0)+b_{i k}^{\prime \prime}(\rho),
$$

with $D(s)=\sum_{i=0}^{n} a_{i 0} s^{i}$ and $N(s)=\sum_{i=0}^{m} b_{i 0} s^{i}$, and

$$
\begin{aligned}
a_{i k}^{\prime}(0)=a_{i k}^{\prime \prime}, & b_{i k}^{\prime}(0)=b_{i k}^{\prime \prime}, \\
a_{i k}^{\prime \prime}(\rho)=\rho a_{i k}^{\prime \prime}(\rho)=o(\rho), & b_{i k}^{\prime \prime}(\rho)=\rho b_{i k}^{\prime \prime}(\rho)=o(\rho) .
\end{aligned}
$$

Thus, an alternative dependent on delay-type asymptotic robust stability condition, which is weaker than those of Theorem 3.2, may be obtained from the above expressions by using Lemma A.2 of the appendix for expanding the denominator quasipolynomial of both the unforced and forced systems with delays (2.1). The key point is the use of Rouché's theorem $[11,16,19]$ in terms of inequalities for each frequency instead of using $H_{\infty}$-norms. Also, a slight variant of Theorem 3.2 may be formulated for asymptotic stability independence of the delays as follows.

Corollary 3.3 (stability independence of the delays). The stability results of items (i), (ii) of Theorem 3.1 also hold independent of delay if the constants $m_{A}, m_{A}^{\prime}$ are redefined dependent on delay with the replacements $q \rightarrow n, A_{i}^{* \prime \prime}(j \omega) \rightarrow A_{i}^{* \prime \prime}(\phi) \omega^{i}, \bar{A}_{i}^{*^{\prime \prime}}(j \omega) \rightarrow \bar{A}_{i}^{\prime \prime}(\phi) \omega^{i}$; and $m_{B}$ and $m_{B}^{\prime}$ of (3.1) are redefined with the replacements $q \rightarrow m, B_{i}^{*^{\prime \prime}}(j \omega) \rightarrow B_{i}^{* \prime \prime}(\phi) \omega^{i}$, $\bar{B}_{i}^{* \prime \prime}(j \omega) \rightarrow \bar{B}_{i}^{\prime \prime}(\phi) \omega^{i}$ and the corresponding simple supreme in (3.1) is taken as double supreme over $\omega \in \mathbb{R}_{0}^{+}$and $\phi \in[0,2 \pi)$. 
Note that, in order for system (2.1) to be globally asymptotically stable independent of delay, it should be stable for zero delay. That means that the auxiliary delay-free system (2.3) has to be globally exponentially stable. Therefore, the stability of (2.3) with transfer function $\bar{P}_{0}(s)$ defined in Lemma 2.1(ii) (2.6), rather than that of (2.2), may be used as necessary condition for the robust stability problem. Thus, close results to Theorems 3.1 and 3.2 are reformulated as follows.

Theorem 3.4 (robust global asymptotic stability from the stability of the delay-free system (2.3)). The subsequent items hold.

(i) Assume that the unforced delay-free system (2.3) is stable with no critical poles. Thus, Theorem 3.1(i) holds for $|\rho| \leq \rho_{0}^{*}=\operatorname{Min}\left(\rho_{0}, 1 / 2 \rho_{0}^{\prime}\right)$, $\rho_{0}$ being a design parameter and $\rho_{0}^{\prime}$ satisfying (3.2) with.

- $\gamma_{0}$ being redefined as the $H_{\infty}$-norm of the unforced delay-free system (2.3) of transfer function $\bar{P}_{0}(s)$.

- The constants (3.1) being redefined from similar expressions for the transfer function of the unforced system (2.1), compared to that of the system (2.3), via (2.6) (i.e., the constants $m_{A}, m_{A}^{\prime}, m_{B}$, and $m_{B}^{\prime}$ being calculated from upper-bounds of the absolute values of $\bar{\Delta}_{A}\left(j \omega, e^{-j \omega h}\right)$ and $\bar{\Delta}_{B}\left(j \omega, e^{-j \omega h}\right)$ for all $\omega \in \mathbb{R}_{0}^{+}$real in (2.6)).

(ii) Assume that the unforced delay-free system (2.3) has no critically stable poles and that it is stabilizable. Assume also that it is stabilized with a controller of transfer function $c(s)$ and that the transfer functions $\bar{P}_{0}(s)$ and $P(s)$ of the unforced systems (2.1) and (2.3) have both the same number $0 \leq n_{u} \leq n$ of unstable poles. Thus, Theorem 3.1(ii) holds for $|\rho| \leq \rho_{0}^{*}=\operatorname{Min}\left(\rho_{0}, 1 / 2 \rho_{0}^{\prime}\right), \rho_{0}$ being a design parameter, and $\rho_{0}^{\prime}$ satisfying (3.3) with the appropriate redefinition of the real constants in (3.1) as in Theorem 3.1(i), with $\gamma$ being redefined as the $H_{\infty}$-norm of the closed-loop transfer function

$$
\bar{T}_{0}(s)=\frac{\bar{P}_{0}(s) c(s)}{1+\bar{P}_{0}(s) c(s)}
$$

(iii) Theorem 3.2 ((i)-(ii)) also holds if the constants $m_{A}, m_{A}^{\prime}$ are redefined with the replacements $q \rightarrow n, A_{i}^{* \prime \prime}(j \omega) \rightarrow A_{i}^{\prime \prime}\left(e^{-j \omega h}\right) \omega^{i}, \bar{A}_{i}^{*^{\prime \prime}}(j \omega) \rightarrow \bar{A}_{i}^{\prime \prime}\left(e^{-j \omega h}\right) \omega^{i}\left(\right.$ for $\bar{\Delta}_{A}\left(j \omega, e^{-j \omega h}\right)$ in (2.6); and $m_{B}$ and $m_{B}^{\prime}$ of (3.1) are redefined with the replacements $q \rightarrow m, B_{i}^{* \prime \prime}(j \omega) \rightarrow$ $B_{i}^{\prime \prime}\left(e^{-j \omega h}\right) \omega^{i}, \bar{B}_{i}^{*^{\prime \prime}}(j \omega) \rightarrow \bar{B}_{i}^{\prime \prime}\left(e^{-j \omega h}\right) \omega^{i}\left(\right.$ for $\bar{\Delta}_{B}\left(j \omega, e^{-j \omega h}\right)$ in $\left.(2.6)\right)$.

Remarks 3.5. (1) Note that the transfer function of (2.1), defined by a quotient of quasipolynomials, may be expanded into two equivalent ways as reflected in (2.4), (2.5) by using polynomial coefficients in $s$ and $\exp (-h s)$, respectively. This fact is used to calculate the relevant constants for guaranteeing stability in two ways, namely, (3.1) for Theorem 3.1 and the modified ones referred to in Theorem 3.2. Two related robust stability conditions are obtained from each of those theorems provided that (2.2) is exponentially stable. The weakest of the above two conditions might be used in practical situations to guarantee stability.

(2) The set of all the alternative conditions for robust global asymptotic stability given by Theorem 3.1, Corollary 3.3, and Theorem 3.4(i) may be checked jointly to conclude that the weakest one is the strongest sufficiency-type robust stability condition of those ones given in this section. 
(3) Since Theorem 3.1 refers to stability independence of the delays, if it holds then the auxiliary delay-free system (2.3) is exponentially stable for the given $\rho$ within some real interval. Since such an interval includes $\rho=0$ zero, then the delay-free system (2.2) is exponentially stable as well.

\section{Some examples}

Example 4.1. Assume again that the parametrization of the system (2.1) with $\sigma=2$ is given by $A_{0}=\left\lfloor\begin{array}{ll}a_{0} & b_{0} \\ c_{0} & d_{0}\end{array}\right\rfloor$ with $\operatorname{Min}\left(\left|a_{0}\right|,\left|d_{0}\right|\right) \geq 1$ being a stability matrix satisfying $\sum_{k=1}^{2}\left\|A_{k}\right\|_{2}<$ $\left(\gamma_{0}^{-1}-\alpha\right) / \rho_{0}^{*}$, some real $\alpha>0$. Thus, the system (2.1) is globally asymptotically stable. Direct calculation yields

$$
\left(s I-A_{0}\right)^{-1}=\left\lfloor\begin{array}{cc}
\frac{1}{s-a_{0}} & \frac{b_{0}}{\left(s-a_{0}\right)\left(s-d_{0}\right)} \\
\frac{c_{0}}{\left(s-a_{0}\right)\left(s-d_{0}\right)} & \frac{1}{s-d_{0}}
\end{array}\right\rfloor,
$$

with

$$
\gamma_{0} \leq \frac{1}{\left|a_{0}\right|}+\frac{1}{\left|d_{0}\right|}+\frac{\left|b_{0}\right|+\left|c_{0}\right|}{\operatorname{Min}^{2}\left(\left|a_{0}\right|,\left|d_{0}\right|\right)} \leq 2\left(1+\frac{\operatorname{Max}\left(\left|b_{0}\right|,\left|c_{0}\right|\right)}{\operatorname{Min}^{2}\left(\left|a_{0}\right|,\left|d_{0}\right|\right)}\right)
$$

for which a sufficient condition is $\gamma_{0}^{-1} \geq(1 / 4)\left(\operatorname{Max}\left(\left|a_{0}\right|,\left|d_{0}\right|\right) / \operatorname{Min}^{2}\left(a_{0}^{2}, d_{0}^{2},\left|c_{0}\right|,\left|d_{0}\right|\right)\right)$. Taking again $A_{0}=\left\lfloor\begin{array}{cc}10 & 30 \\ -9 & -25\end{array}\right\rfloor$, the following stability results hold.

(1) The system is globally asymptotically stable independent of delay from Theorem 3.1 and its characteristic roots lie in $\operatorname{Re} s \leq-0.1$ if $\rho_{0}^{\prime} \leq 0.291$ which is achieved, for instance, with delayed dynamics given by $A_{1}=\left\lfloor\begin{array}{cc} \pm 0.04 & b_{1} \\ 0 & \pm 0.017\end{array}\right\rfloor, A_{2}=\left\lfloor\begin{array}{cc} \pm 0.28 & 0 \\ c_{2} & \pm 0.2\end{array}\right\rfloor$ for arbitrary finite $b_{1}$ and $c_{2}$.

(2) The system is globally asymptotically stable dependent on the delay from Theorem 3.2 with the same $A_{0}$ as above if $\rho=1.034$ for $h \in\left[0, h_{M}\right]$ with $h_{M}=10^{3} \ln 1.034 / 2=$ 16.717. This is accomplished, for instance, by the parametrization

$$
A_{1}=\left\lfloor\begin{array}{cc} 
\pm 0.1 & b_{1} \\
0 & \pm 0.11
\end{array}\right\rfloor ; \quad A_{2}=\left\lfloor\begin{array}{cc} 
\pm 0.206 & 0 \\
c & \pm 0.09
\end{array}\right\rfloor .
$$

(3) The system is stable dependent on the delay from Theorem 3.2 with the same $A_{0}$ as above and $A_{1}=\left\lfloor\begin{array}{cc} \pm 0.045 & b_{1} \\ 0 & \pm 0.044\end{array}\right\rfloor ; A_{2}=\left\lfloor\begin{array}{cc} \pm 0.045 & 0 \\ c_{1} & \pm 0.045\end{array}\right\rfloor$, with $h \in\left[0, h_{M}\right\rfloor$ with $h_{M}=10^{3} \ln 2.3 / 2$ $=416.454$.

Example 4.2. Assume that the delay-free dynamics of the system (2.1) with $n=3$ is given by the pair $\left(A_{0}, b\right)$ with $A_{0}=\left[\begin{array}{ccc}a_{1} & a_{2} & a_{3} \\ 1 & 0 & 0 \\ 0 & 1 & 0\end{array}\right]$ and $b^{T}=(1,0,0)$ which is controllable, so that it is stabilizable as well if $a_{k} \neq 0$ for $k=1,2,3$. The open-loop characteristic polynomial of the delay-free system (2.2) is $p_{o}(s)=s^{2}-a_{1} s^{2}-a_{2} s-a_{3}$. Assume that the closed-loop characteristic polynomial is suited to be $p_{c}(s)=\left(s-\beta_{1}\right)\left(s-\beta_{2}\right)\left(s-\beta_{3}\right)=s^{2}-\alpha_{1} s^{2}-\alpha_{2} s-\alpha_{3}$, where $\operatorname{Re} \beta_{k}<0(k=1,2,3)$, and

$$
\alpha_{1}=\beta_{1}+\beta_{2}+\beta_{3} ; \quad \alpha_{2}=-\left[\beta_{1} \beta_{2}+\left(\beta_{1}+\beta_{2}\right) \beta_{3}\right] ; \quad \alpha_{3}=\beta_{1} \beta_{2} \beta_{3} .
$$


Since the delay-free closed-loop dynamics under linear state feedback is defined by the matrix

$$
A_{c}=A_{0}+b K^{T}=\left[\begin{array}{ccc}
a_{1}-k_{1} & a_{2}-k_{2} & a_{3}-k_{3} \\
1 & 0 & 0 \\
0 & 1 & 0
\end{array}\right] \text {, }
$$

then the controller gain matrix components are

$$
\begin{aligned}
& k_{1}=a_{1}-\left(\beta_{1}+\beta_{2}+\beta_{3}\right) ; \\
& k_{2}=a_{2}+\beta_{1} \beta_{2}+\left(\beta_{1}+\beta_{2}\right) \beta_{3} ; \\
& k_{3}=a_{3}-\beta_{1} \beta_{2} \beta_{3} .
\end{aligned}
$$

The $H_{\infty}$-gain of $\left(A_{0}+b K^{T}\right)$ is $\gamma_{0 c} \leq K_{0} / \prod_{k=1}^{3}\left|\operatorname{Re} \beta_{k}\right| \leq K_{0} / \rho_{0}^{3}$ for some real constant $K_{0} \geq 1$ with the design parameter $\rho_{0}$ being chosen as the negative stability abscissa of $A_{c}$. Thus, the closed-loop system is globally asymptotically stable dependent on delay for any set or point delays whose dynamics satisfies $\prod_{k=1}^{3}\left|\operatorname{Re} \beta_{k}\right|>K_{0} \sum_{k=1}^{\sigma}\left\|A_{k}\right\|_{2}$ and stable and all delays $h \in\left[0, \ln k_{m} / \alpha\right]$ (Theorem 3.2) for some $k_{m} \geq 1$ provided that $\prod_{k=1}^{3}\left|\operatorname{Re} \beta_{k}\right|>$ $K_{0}\left(k_{m} \sum_{k=1}^{\sigma}\left\|A_{k}\right\|_{2}+\alpha\right)$. Any controller gain matrix $K$ which generated closed-loop poles at positions $\operatorname{Re} \beta_{k} \leq-\rho_{0}$ gives asymptotic stability independent of delay under conditions $\rho_{0}^{3}>K_{0} \sum_{k=1}^{\sigma}\left\|A_{k}\right\|_{2}$ and $\rho_{0}^{3}>K_{0}\left(\sum_{k=1}^{\sigma}\left\|A_{k}\right\|_{2}+\alpha\right)$, respectively. If $\left(A_{0}, b\right)$ is not in the controllable canonical form, then Ackerman's formula (see, for instance, [11]) may be used to calculate the gain matrix

$$
K=[0,0,1]\left[b, A_{0} b, A_{0}^{2} b\right]^{-1}\left[A^{3}-\alpha_{1} A^{2}-\alpha_{2} A-\alpha_{3} I\right] \alpha_{c}(A),
$$

where $\alpha_{c}(A)=-\sum_{k=0}^{3} \alpha_{k} s^{3-k}\left(\alpha_{0}=-1\right)$ if the objective closed-loop characteristic polynomial is $p_{c}(s)=-\sum_{k=0}^{3} \alpha_{i} s^{3-i}$. Now, assume that $\left(A_{0}, b\right)$ is stabilizable with $A_{0}=\left[\begin{array}{cccc}a_{1} & a_{2} & a_{3} \\ 1 & 0 & 0 \\ 0 & 1 & 0\end{array}\right]$ and $b^{T}=(1,0,0)$ so that $D(s)=p_{0}(s)=\operatorname{Det}\left(s I-A_{0}\right)=\left(s^{2}-a_{1} s-a_{2}\right)(s-\beta)$, where $s=\beta$ is a stable root which cannot be relocated via linear state-feedback. Now, the suited strictly Hurwitzian characteristic closed-loop polynomial is $p_{c}(s)=\left(s-\beta_{1}\right)\left(s-\beta_{2}\right)(s-\beta)$ which is achieved through linear state-feedback by using the controller gain components $k_{1}=$ $a_{1}-\left(\beta_{1}+\beta_{2}+\beta\right) ; k_{2}=a_{2}+\beta_{1} \beta_{2}+\left(\beta_{1}+\beta_{2}\right) \beta ; k_{3}=a_{3}-\beta_{1} \beta_{2} \beta$. The conditions for global asymptotic closed-loop stabilization independent of delay under the presence of $\sigma$ point delays are $|\operatorname{Re} \beta|>K_{0}\left(\sum_{k=1}^{\sigma}\left\|A_{k}\right\|_{2} / \prod_{k=1}^{2}\left|\operatorname{Re} \beta_{k}\right|\right)$.

Example 4.3. Now, the stability conditions are manipulated for all frequencies, [14], under the guidelines of Lemma A.2 in the appendix (see also $[9,14]$ ), rather than in terms of $H_{\infty}$. Consider the delay-free third-order system of characteristic polynomial given by

$$
p_{0}(s)=s^{3}+a_{1}^{*} s+a_{2}^{*} s+a_{3}^{*}
$$

which suffers a perturbation caused by delayed dynamics given by

$$
\Delta p(s)=p(s)-p_{0}(s)=\rho\left[\left(1+\Delta a_{1} s\right) e^{-h s}+\left(\Delta a_{21}+\Delta a_{22} s\right) e^{-2 h s}\right] .
$$


Direct calculus yields

$$
\begin{gathered}
p_{0}(j \omega)=\left(a_{3}^{*}-a_{1}^{*} \omega^{2}\right)+j \omega\left(a_{2}^{*}-\omega^{2}\right) ; \\
\Delta p(j \omega)=\rho e^{-j h \omega}\left\{\left(1+j \Delta a_{1} \omega\right)+e^{-j h \omega}\left(\Delta a_{21}+j \Delta a_{22} \omega\right)\right\},
\end{gathered}
$$

so that

$$
\begin{gathered}
\left|p_{0}(j \omega)\right|^{2}=\left(a_{3}^{*}-a_{1}^{*} \omega^{2}\right)^{2}+\left(a_{2}^{*} \omega-\omega^{3}\right)^{2} ; \\
|\Delta p(j \omega)|^{2} \leq 2 \rho^{2}\left[\left(1+\Delta a_{1}^{2} \omega^{2}\right)+\left(\Delta a_{21}^{2}+\Delta a_{22}^{2} \omega^{2}\right)\right] .
\end{gathered}
$$

The condition $|\Delta p(j \omega)|<\left|p_{0}(j \omega)\right|$, for all $\omega \in \mathbb{R}_{+}^{0}$, becomes

$$
\begin{aligned}
F(\omega):= & \omega^{6}+\left(a_{1}^{* 2}-2 a_{2}^{*}\right) \omega^{4}+\left[a_{2}^{* 2}-2 a_{1}^{*} a_{3}^{*}-2 \rho^{2}(h)\left(\Delta a_{1}^{2}+\Delta a_{22}^{2}\right)\right] \omega^{2} \\
& +\left[a_{3}^{* 2}-2 \rho^{2}(h)\left(1+\Delta a_{21}^{2}\right)\right]>0
\end{aligned}
$$

for all $\omega \in \mathbb{R}_{+}^{0}$. If the stability conditions of Theorem 3.1 are relaxed according to Lemma A.2, then all the coefficients of $F(\omega)$ have to be positive. Thus, the system of characteristic equation $p(s)=0$ is globally asymptotically stable if the subsequent conditions hold.

(1) $p_{0}(s)$ is strictly Hurwitzian. According to the Routh-Hurwitz criterion, this holds if and only if $a_{i}^{*}>0(i=1,2,3)$ and $a_{3}^{*}<a_{1}^{*} a_{2}^{*}$.

(2) $F(\omega)>0$, for all $\omega \in \mathbb{R}_{0}^{+}$, with all the coefficients being positive, that is, $a_{1}^{*}>2 a_{2}^{*}$; $a_{2}^{* 2}>2 a_{1}^{*} a_{3}^{*}\left(\Rightarrow a_{1}^{*}>\operatorname{Max}\left(\sqrt{2 a_{2}^{*}}, \sqrt[3]{2 a_{3}^{*}}, a_{3}^{*} / a_{2}^{*}\right)\right.$ according to the two above constraints $)$ if

$$
\rho^{2}<\operatorname{Min}\left(\frac{a_{3}^{*^{2}}}{2\left(1+\Delta a_{21}^{2}\right)}, \frac{a_{2}^{*^{2}}-2 a_{1}^{*} a_{3}^{*}}{2\left(\Delta a_{1}^{2}+\Delta a_{22}^{2}\right)}\right) \text {. }
$$

Some coefficients of $F(\omega)$ may be allowed to be nonpositive and compensated with other being positive over the whole frequency range $[0, \infty)$. Note that $a_{1}^{*^{2}}-2 a_{2}^{*}>0$ and $a_{3}^{*^{2}}-$ $\rho^{2}(h)\left(1+\Delta a_{21}^{2}\right)>0$ in order that $F(\infty)>0$ and $F(0)>0$, respectively. The coefficient of $\omega^{2}$ may be nonpositive with $a_{2}^{*^{2}} \leq 2 a_{1}^{*} a_{3}^{*}$. Thus, it is obvious that for $\omega \in[0,1]$,

$$
\begin{aligned}
F(\omega) & \geq a_{3}^{*^{2}}-\rho^{2}\left(1+\Delta a_{21}^{2}\right)-\left(\left|a_{2}^{*^{2}}-2 a_{1}^{*} a_{3}^{*}\right|+2 \rho^{2}(h)\left(\Delta a_{1}^{2}+\Delta a_{22}^{2}\right)\right) \omega^{2} \\
& \geq a_{3}^{*^{2}}-\left|a_{2}^{*^{2}}-2 a_{1}^{*} a_{3}^{*}\right|+2 \rho^{2}(h)\left(1+\Delta a_{1}^{2}+\Delta a_{21}^{2}+\Delta a_{22}^{2}\right)>0
\end{aligned}
$$

which is guaranteed if $\rho^{2}<\left(a_{3}^{*^{2}}-\left|a_{2}^{*}-2 a_{1}^{*} a_{3}^{*}\right|\right) / 2\left(1+\Delta a_{1}^{2}+\Delta a_{21}^{2}+\Delta a_{22}^{2}\right)$ provided that $a_{3}^{*^{2}}>\left|a_{2}^{*}-2 a_{1}^{*} a_{3}^{*}\right|$. For all real $\omega \geq 1$, note that

$$
F(\omega) \geq 1+\left(a_{1}^{*^{2}}-2 a_{2}^{*}\right)+\left(a_{3}^{*^{2}}-\left|a_{2}^{*^{2}}-2 a_{1}^{*} a_{3}^{*}\right|\right)-2 \rho^{2}(h)\left(1+\Delta a_{1}^{2}+\Delta a_{21}^{2}+\Delta a_{22}^{2}\right)>0,
$$

which is guaranteed if $\rho^{2}<\left(1+a_{3}^{*^{2}}+\left(a_{1}^{*}-2 a_{2}^{*}\right)-\left|a_{2}^{*}-2 a_{1}^{*} a_{3}^{*}\right|\right) / 2\left(1+\Delta a_{1}^{2}+\Delta a_{21}^{2}+\Delta a_{22}^{2}\right)$ which is weaker than the condition obtained for $\omega \in[0,1]$, so that it has not been taken into account. Thus, the set of conditions for stability are either Conditions 4.4, 4.5 or Conditions 4.4-4.6 below. 
Conditions 4.4 .

$$
a_{i}^{*}>0 \quad(i=1,2,3) ; \quad a_{1}^{*^{2}} \geq 2 a_{2}^{*} ; \quad a_{1}^{*}>\frac{a_{3}^{*}}{a_{2}^{*}}\left(\Longrightarrow a_{1}^{*}>\operatorname{Max}\left(\frac{a_{3}^{*}}{a_{2}^{*}}, \sqrt{2 a_{2}^{*}}\right)\right) .
$$

Conditions 4.5. If $a_{2}^{*^{2}}>2 a_{1}^{*} a_{3}^{*}\left(\Rightarrow a_{1}^{*}>\operatorname{Max}\left(\sqrt{2 a_{2}^{*}}, \sqrt[3]{2 a_{3}^{*}}, a_{3}^{*} / a_{2}^{*}\right)\right)$, then

$$
\rho^{2}<\frac{1}{2} \operatorname{Min}\left(\frac{a_{3}^{*^{2}}}{1+\Delta a_{21}^{2}}, \frac{a_{2}^{*^{2}}-2 a_{1}^{*} a_{3}^{*}}{\Delta a_{1}^{2}+\Delta a_{22}^{2}}\right) .
$$

Conditions 4.6. If $a_{2}^{*^{2}} \leq 2 a_{1}^{*} a_{3}^{*}$, then

$$
\rho^{2}<\frac{1}{2} \operatorname{Min}\left(\frac{a_{3}^{*^{2}}}{1+\Delta a_{21}^{2}}, \frac{a_{3}^{*^{2}}-\left|a_{2}^{*^{2}}-2 a_{1}^{*} a_{3}^{*}\right|}{1+\left(\Delta a_{1}^{2}+\Delta a_{21}^{2}+\Delta a_{22}^{2}\right)}\right) .
$$

Now, consider

$$
\begin{aligned}
\Delta p(j \omega)=\rho\left\{\cos \varphi+\Delta a_{1} \omega \sin \varphi+\Delta a_{21} \cos 2 \varphi+\omega \Delta a_{22} \sin 2 \varphi\right. \\
\left.+j\left[\left(\Delta a_{1} \cos \varphi+\Delta a_{22} \cos 2 \varphi\right)-j\left(\sin \varphi+\Delta a_{21} \sin 2 \varphi\right)\right]\right\},
\end{aligned}
$$

with $\varphi=h \omega$ (see Corollary 3.3) so that after grouping terms, the system is globally asymptotically stable if $p_{0}(s)$ is strictly Hurwitzian, that is, all its zeros lie in $\operatorname{Re} s<0$, and

$$
\begin{aligned}
|\Delta p(j \omega)|^{2}= & \rho^{2}\left\{1+\Delta a_{21}^{2}+\left(\Delta a_{1}^{2}+\Delta a_{22}^{2} \omega^{2}\right)+4\left(\Delta a_{21}+\Delta a_{1} \Delta a_{22} \omega^{2}\right)\left(\cos \varphi-\cos ^{3} \varphi\right)\right. \\
& \left.+2\left(\Delta a_{21}+\Delta a_{1} \Delta a_{22} \omega^{2}\right)\left(2 \cos ^{3} \varphi-\cos \varphi\right)\right\} \\
\leq & \rho^{2}\left\{1+\Delta a_{21}^{2}+\left(\Delta a_{1}^{2}+\Delta a_{22}^{2}\right) \omega^{2}+2\left|\Delta a_{21}+\Delta a_{1} \Delta a_{22} \omega^{2}\right|\right\} \\
< & \left|p_{0}(j \omega)\right|^{2}=\omega^{6}+\left(a_{1}^{*^{2}}-2 a_{2}^{*}\right) \omega^{4}+\left(a_{2}^{*^{2}}-2 a_{1}^{*} a_{3}^{*}\right) \omega^{2}+a_{3}^{*^{2}},
\end{aligned}
$$

namely, if

$$
\begin{aligned}
\omega^{6} & +\left(a_{1}^{*^{2}}-2 a_{2}^{*}\right) \omega^{4}+\left[a_{2}^{*^{2}}-2 a_{1}^{*} a_{3}^{*}-\rho^{2}\left(\left|\Delta a_{1}\right|+\left|\Delta a_{22}\right|\right)^{2}\right] \omega^{2} \\
& +\left[a_{3}^{*^{2}}-\rho^{2}\left(1+\left|\Delta a_{21}\right|\right)^{2}\right]>0, \quad \forall \omega \in \mathbb{R}_{0}^{+},
\end{aligned}
$$

which holds if $|\rho|<\rho_{0}^{*}:=\operatorname{Min}\left(\left|a_{3}^{*}\right| /\left(1+\left|\Delta a_{21}\right|\right),\left|a_{2}^{*^{2}}-2 a_{1}^{*} a_{3}^{*}\right| /\left(\left|\Delta a_{1}\right|+\left|\Delta a_{22}\right|\right)\right)$, which guarantees the robust global asymptotic stability of (2.1) independent of the delays.

\section{Conclusions}

This paper has addressed sufficiency-type robust stability conditions for linear timeinvariant systems with constant point delays. The delayed dynamics is compared for robust stability purposes with that of two auxiliary delay-free systems which are, respectively, obtained by either neglecting the delayed dynamics or by considering that the delays are zero. The stability theorems are derived in the frequency domain for systems 
whose delay-free versions are stable. They are based on the use of Rouché's theorem on location of the zeros of two analytical functions in prescribed domains and the maximum module principle for analytic functions. The sufficiency-type asymptotic stability conditions obtained from the stability criteria may be dependent or independent of the delays. Some examples presented through the paper illustrate the application of the above-mentioned stability criteria.

Some of the given results are of "independent of delays" type while others are of "dependent on delays" type and both situations have been addressed in this manuscript. It has been proved that the delayed system is asymptotically stable if the inverse of the $H_{\infty}$ gain of the delay-free dynamical system is sufficiently large compared to the size (in terms of norms) of the delayed dynamics if the delay-free auxiliary system is exponentially stable. A set of complementary results has been devoted to investigate the stability properties under linear delay-free feedback. It is required that the open-loop system be stabilizable for state linear feedback.

\section{Appendix}

Proof of Lemma 2.1. (i) From direct calculus, the transfer function of the system (2.1) is a rational transcendent complex function defined by

$$
P(s):=c^{T} \operatorname{Adj}\left(s I-\mathbf{A}_{0}-\rho \sum_{i=1}^{\sigma} \mathbf{A}_{\mathbf{i}} e^{-i h s}\right)^{-1} \quad b=\frac{B\left(s, e^{-h s}\right)}{A\left(s, e^{-h s}\right)},
$$

where the denominator quasipolynomial is

$$
\begin{aligned}
A\left(s, e^{-h s}\right): & =\operatorname{det}\left(s I-\mathbf{A}_{\mathbf{0}}-\rho \sum_{i=1}^{\sigma} \mathbf{A}_{\mathbf{i}} e^{-i h s}\right)=\sum_{i=0}^{n} A_{i}\left(e^{-h s}\right) s^{i}=\sum_{k=0}^{q} A_{k}^{*}(s) e^{-k h s} \\
& =\sum_{i=0}^{n} \sum_{k=0}^{q} a_{i k} s^{i} e^{-k h s}=D(s)+\sum_{i=0}^{n} \sum_{k=1}^{q} a_{i k} s^{i} e^{-k h s}
\end{aligned}
$$

and the numerator quasipolynomial is

$$
\begin{aligned}
B\left(s, e^{-h s}\right): & =c^{T} \operatorname{Adj}\left(s I-\mathbf{A}_{0}-\rho \sum_{i=1}^{\sigma} \mathbf{A}_{\mathbf{i}} e^{-i h s}\right) b=\sum_{i=0}^{m} B_{i}\left(e^{-h s}\right) s^{i} \\
& =\sum_{k=0}^{q} B_{k}^{*}(s) e^{-k h s}=\sum_{i=0}^{m} \sum_{k=0}^{q_{1}} b_{i k} s^{i} e^{-k h s}
\end{aligned}
$$

with $q_{1} \leq q \leq n \sigma$, and polynomials

$$
A_{i}^{*}(s)=\sum_{k=0}^{n} a_{k i} s^{k} ; \quad A_{\ell}\left(e^{-h s}\right)=\sum_{k=1}^{q} a_{\ell k} e^{-k h s}
$$


for $i=0,1, \ldots, n ; l=0,1, \ldots, q_{1}$, with $D(s):=\operatorname{det}\left(s I-\mathbf{A}_{0}\right)=A_{0}^{*}(s)=\sum_{k=0}^{n} a_{k 0} s^{k}$ being the characteristic polynomial of the delay-free system (2.2) and

$$
B_{i}^{*}(s)=\sum_{k=0}^{m} b_{k i} s^{k} ; \quad B_{\ell}\left(e^{-h s}\right)=\sum_{k=1}^{q_{1}} b_{\ell k} e^{-k h s}
$$

for $i=0,1 \ldots, m ; l=0,1, \ldots, q$. By zeroing the corresponding exceeding parameters, if necessary, it may be always assumed in the following with no loss in generality that $q_{1}=q=n \sigma$. The polynomials $A_{i}(s)(i=0,1, \ldots, n)$ and $B_{k}(s)(k=0,1, \ldots, q)$ may be calculated recursively as follows:

$$
\begin{gathered}
A_{n-i}\left(e^{-h s}\right)=-\frac{1}{i} \operatorname{tr}\left\lfloor S_{n-i}\left(e^{-h s}\right)\left(\mathbf{A}_{\mathbf{0}}+\rho \sum_{k=1}^{q} \mathbf{A}_{\mathbf{k}} e^{-k h s}\right)\right\rfloor, \\
S_{n-i}\left(e^{-h s}\right)=S_{n-i+1}\left(e^{-h s}\right)\left(\mathbf{A}_{\mathbf{0}}+\rho \sum_{k=1}^{q} \mathbf{A}_{\mathbf{k}} e^{-k h s}\right)+A_{n-i+1}\left(e^{-h s}\right) I
\end{gathered}
$$

for $i=1,2, \ldots, n$ with initial value $S_{n-1}\left(e^{-h s}\right)=I$, so that

$$
\begin{aligned}
S_{-1}\left(e^{-h s}\right)=S_{0}\left(e^{-h s}\right)\left(\mathbf{A}_{0}+\rho \sum_{k=1}^{q} \mathbf{A}_{\mathbf{k}} e^{-k h s}\right)+A_{0}\left(e^{-h s}\right) I=0, \\
B\left(s, e^{-h s}\right)=c^{T}\left[\mathbf{A}^{n-1}\left(e^{-h s}\right)+\left(s+A_{n-1}^{*}(s)\right) \mathbf{A}^{n-2}\left(e^{-h s}\right)+\cdots+A_{1}^{*}(s) I\right] b \\
=c^{T}\left(\sum_{i=0}^{n} S_{i}\left(e^{-h s}\right) s^{i}\right) b
\end{aligned}
$$

where $\mathbf{A}\left(e^{-h s}\right)=\sum_{k=0}^{q} \mathbf{A}_{\mathbf{k}} e^{-k h s}$ and the maximum degrees of the polynomials and matrix polynomial entries in (A.8) are $m \leq n-1$ in $s$ and $q$ in $e^{-h s}$, respectively. The transfer function (A.1) becomes

$$
P(s)=\frac{c^{T}\left(\sum_{i=0}^{n} S_{i}\left(e^{-h s}\right) s^{i}\right) b}{\sum_{i=0}^{n} A_{i}\left(e^{-h s}\right)}=\frac{\sum_{i=0}^{m} B_{i}\left(e^{-h s}\right) s^{i}}{\sum_{i=0}^{n} A_{i}\left(e^{-h s}\right) s^{i}}=\frac{\sum_{k=0}^{q} B_{k}^{*}(s) e^{-k h s}}{\sum_{k=0}^{n} B_{k}^{*}(s) e^{-k h s}}
$$

which can be calculated explicitly via (A.6)-(A.8). If $\rho=0$ then (2.1) is described by the unforced delay-free auxiliary system of transfer function

$$
P_{0}(s)=\frac{c^{T} \operatorname{Adj}(s I-A) b}{D(s)}=\frac{c^{T}\left(\sum_{i=0}^{n} S_{i}^{0} s^{i}\right) b}{\sum_{i=0}^{n} A_{i}^{0} s^{i}}=\frac{N(s)}{D(s)}
$$

where $D(s)=\operatorname{det}\left(s I-\mathbf{A}_{\mathbf{0}}\right)$ is its characteristic polynomial and $S_{i}^{0}$ and $A_{i}^{0}$ are real matrices and real numbers, respectively, defined recursively by

$$
A_{n-i}^{0}=-\frac{1}{i} \operatorname{tr}\left[S_{n-i}^{0} \mathbf{A}_{0}\right] ; \quad S_{n-i}^{0}=S_{n-i+1}^{0} A_{0}+A_{n-i+1}^{0} I ; i=1,2, \ldots, n,
$$


with initial value $S_{n-1}^{0}=I$ so that $S_{-1}^{0}=S_{0}^{0} \mathbf{A}_{0}+A_{0}^{0} I=0$. Substituting (A.10) into (A.6) via (A.4), (A.5), one gets for $i=1,2, \ldots, n$,

$$
\begin{aligned}
A_{n-i}\left(e^{-h s}\right)=A_{n-i}^{0}+\rho A_{n-i}^{\prime}\left(e^{-h s}\right)=A_{n-i}^{0}+\rho A_{n-i}^{\prime \prime}\left(e^{-h s}\right)+o(\rho), \\
B_{n-i}\left(e^{-h s}\right)=c^{T}\left(\mathbf{A}_{\mathbf{0}}+\rho \sum_{k=1}^{n} \mathbf{A}_{\mathbf{k}} e^{-k h s}\right)^{\ell} b \\
=c^{T}\left(A_{0}^{\ell}+\rho \sum_{k=1}^{n}\left(\begin{array}{l}
\ell \\
1
\end{array}\right) \mathbf{A}_{\mathbf{0}}^{\ell-1} \mathbf{A}_{\mathbf{k}}^{\ell} e^{-h s}+\rho^{2} \sum_{i=2}^{\ell} \sum_{k=1}^{n} \rho^{i-2}\left(\begin{array}{l}
\ell \\
k
\end{array}\right) \mathbf{A}_{\mathbf{0}}^{\ell-\mathbf{k}} \mathbf{A}_{\mathbf{k}}^{\ell} e^{-i k h s}\right) b \\
=B_{n-i}^{0}+\rho B_{n-i}^{\prime}\left(e^{-h s}\right)=B_{n-i}^{0}+\rho B_{n-i}^{\prime \prime}\left(e^{-h s}\right)+o(\rho),
\end{aligned}
$$

with $A_{i}^{0}=a_{i 0} ; B_{k}^{0}=b_{k 0}$ for $i=0,1, \ldots, n$ and $k=0,1, \ldots, m$, since (A.2b) may be rewritten as

$$
\begin{aligned}
A\left(s, e^{-h s}\right) & =\operatorname{det}\left(s I-\mathbf{A}_{\mathbf{0}}-\rho \sum_{i=1}^{n} \mathbf{A}_{\mathbf{i}} e^{-i h s}\right)=A_{0}^{*}(s) \operatorname{det}\left(I-\rho\left(s I-\mathbf{A}_{\mathbf{0}}\right)^{-1} \sum_{i=1}^{n} \mathbf{A}_{\mathbf{i}} e^{-i h s}\right) \\
& =D(s)\left(1-\rho \operatorname{tr}\left(s I-\mathbf{A}_{\mathbf{0}}\right)^{-1} \sum_{i=1}^{n} \mathbf{A}_{\mathbf{i}} e^{-i h s}\right)+o(\rho)
\end{aligned}
$$

for all complex $s$, such that $D(s)=A_{0}^{*}(s) \neq 0$ which is the case for all the infinitely many characteristic roots of (2.1) for all nonzero real $\rho$ since, otherwise, the characteristic equation of (2.1) would be $D(s)=0$ which is impossible for $\rho \neq 0$. In the above expressions, $o(\rho)$ ("small-o" Landau's notation) applies for a complex function of $s$ which is bounded for all finite $\rho$ and $s$ and tends to zero as $\rho \rightarrow 0$. Now, from (A.11), (A.12), the proof of (i) follows with

$$
\begin{gathered}
\Delta_{A}\left(s, e^{-h s}\right)=\sum_{i=1}^{n} A_{i}^{\prime}\left(e^{-h s}\right) s^{i}=\sum_{i=1}^{q} A_{i}^{*^{\prime}}(s) e^{-i h s}, \\
\Delta_{B}\left(s, e^{-h s}\right)=\sum_{i=1}^{n} B_{i}^{\prime}\left(e^{-h s}\right) s^{i}=\sum_{i=1}^{q} B_{i}^{*^{\prime}}\left(e^{-h s}\right) e^{-i h s},
\end{gathered}
$$

where

$$
\begin{gathered}
A_{i}^{*^{\prime}}(s)=A_{i}^{*^{\prime \prime}}(s)+\rho \bar{A}_{i}^{*^{\prime \prime}}(s) ; \quad i=1,2, \ldots, q, \\
B_{i}^{*^{\prime}}(s)=B_{i}^{*^{\prime \prime}}(s)+\rho \bar{B}_{i}^{*^{\prime \prime}}(s) ; \quad i=1,2, \ldots, q, \\
A_{i}^{\prime}\left(e^{-h s}\right)=A_{i}^{\prime \prime}\left(e^{-h s}\right)+\rho \bar{A}_{i}^{\prime \prime}\left(e^{-h s}\right) ; \quad i=1,2, \ldots, n, \\
B_{i}^{\prime}\left(e^{-h s}\right)=B_{i}^{\prime \prime}\left(e^{-h s}\right)+\rho \bar{B}_{i}^{\prime \prime}\left(e^{-h s}\right) ; \quad i=1,2, \ldots, m,
\end{gathered}
$$


for all real $\rho$ with the coefficients of $\bar{A}_{i}^{*^{\prime \prime}}(\cdot), \bar{A}_{i}^{\prime \prime}(\cdot)$ and $\bar{B}_{i}^{*^{\prime \prime}}(\cdot), \bar{B}_{i}^{\prime \prime}(\cdot)$ being, in general, dependent on powers of $\rho$ varying from $\rho^{1}$ up till $\rho^{n}$ and $o(\rho)$ being a bounded complex function for all finite complex $s$ and real $\rho, o(\rho) \rightarrow 0$ as $\rho \rightarrow 0$ and $D(s)=A_{0}^{*}(s)=\operatorname{det}(s I-$ $\left.\mathbf{A}_{\mathbf{0}}\right)$ and $N(s)=B_{0}^{*}(s)$ for $\rho=0$.

(ii) The proof becomes direct by rewriting (A.12) for any complex $s$ such that $\bar{D}(s) \neq 0$ as

$$
\begin{aligned}
A\left(s, e^{-h s}\right) & =\operatorname{det}\left(s I-\mathbf{A}_{\mathbf{0}}-\rho \sum_{i=1}^{n} \mathbf{A}_{\mathbf{i}}-\rho \sum_{i=1}^{n} \mathbf{A}_{\mathbf{i}}\left(e^{-i h s}-1\right)\right) \\
& =\bar{D}(s) \operatorname{det}\left(I-\rho\left(s I-\mathbf{A}_{\mathbf{0}}-\rho \sum_{i=1}^{n} \mathbf{A}_{\mathbf{i}}\right)^{-1} \sum_{i=1}^{n} \mathbf{A}_{\mathbf{i}}\left(e^{-i h s}-1\right)\right) \\
& =\bar{D}(s)\left(1-\rho \operatorname{tr}\left(s I-\mathbf{A}_{\mathbf{0}}-\rho \sum_{i=1}^{n} \mathbf{A}_{\mathbf{i}}\right)^{-1} \sum_{i=1}^{n} \mathbf{A}_{\mathbf{i}}\left(e^{-i h s}-1\right)\right)+o(\rho)
\end{aligned}
$$

and a parallel identity to (A.8) for $B\left(s, e^{-h s}\right)$.

Remark A.1. It turns out from (A.13) that the transfer function of Lemma 2.1 may be also equivalently calculated from the polynomials $A_{(\cdot)}^{*^{\prime}}(s)$ and $B_{(\cdot)}^{*^{\prime}}(s)$ since

$$
\begin{aligned}
B\left(s, e^{-h s}\right) & =B_{0}^{*}(s)+\rho \sum_{i=1}^{n} \sum_{k=1}^{q} b_{i k}^{\prime}(\rho) s^{i} e^{-k h s}=B_{0}^{*}(s)+\rho \sum_{i=1}^{n} B_{i}^{\prime}\left(e^{-h s}\right) s^{i} \\
& =B_{0}^{*}(s)+\rho \sum_{i=1}^{q} B_{i}^{*^{\prime}}(s) e^{-i h s}=D(s)+\rho \Delta_{b}(s), \\
A\left(s, e^{-h s}\right) & =A_{0}^{*}(s)+\rho \sum_{i=1}^{n} \sum_{k=1}^{q} a_{i k}^{\prime}(\rho) s^{i} e^{-k h s}=D(s)+\rho \sum_{i=1}^{n} A_{i}^{\prime}\left(e^{-h s}\right) s^{i} \\
& =D(s)+\rho \sum_{i=1}^{q} A_{i}^{*^{\prime}}(s) e^{-i h s}=D(s)+\rho \Delta_{a}(s) .
\end{aligned}
$$

Thus, the transfer function of (2.1) may be also equivalently expressed as $T(s)=$ $c^{T}\left(\sum_{i=0}^{q} S_{i}^{*}(s) e^{-i h s}\right) b / \sum_{i=0}^{q} A_{i}^{*}(s) e^{-i h s}$, where $S_{i}^{*}(s), A_{i}^{*}(s)$, and $A_{i}^{*^{\prime}}(s)(i=1,2, \ldots, q)$ are polynomials defined similarly to those in (A.6) by replacing $e^{-h s}$ with $s$ accordingly to the definitions in (A.4), (A.5).

Proof of Theorem 3.1. (i) Assume that for any positive real arbitrary $\rho_{0},|\rho| \leq \rho_{0}$. Note that if the system (2.1) is considered as a perturbed one with respect to a nominal proper exponentially stable system (2.2), then (2.1) is globally asymptotically stable if $|\rho| \leq 1 / \rho_{0}^{\prime}$, with $\rho_{0}^{\prime}$ being defined in (3.2) and in general dependent on $\rho_{0}$ for real constants $m_{A}$ and $m_{A}^{\prime}$ being defined in (3.1) from $\Delta_{A}\left(j \omega, e^{-j \omega h}\right)$ and $\Delta_{B}\left(j \omega, e^{-j \omega h}\right)$ in Lemma 2.1(i), since

$$
|\rho|\left\lfloor\left|\frac{A^{*^{\prime}}(j \omega)}{D(j \omega)}\right|\right\rfloor \leq|\rho| \rho_{0}^{\prime}<1 \Longleftrightarrow|\rho|\left|A^{*^{\prime}}(j \omega)\right|<|D(j \omega)|
$$


for all $\omega \in \mathbb{R}_{0}^{+}$implies from Rouché's theorem, $[11,16,19]$, that $A\left(s, e^{-h s}\right)$ and $D(s)$ have the same number of unstable zeros; that is, none since the unforced system (2.2) is exponentially stable. Note that no test in (A.16) is necessary for negative frequencies since $|G(-j \omega)|=|G(j \omega)|$ for any transfer function $G(s)$. Thus, if $|\rho| \rho_{0}^{\prime}<1$, the proof follows directly. Otherwise, the proof follows as well since the inequalities in (A.16) are fulfilled for $|\rho| \leq \operatorname{Min}\left(\rho_{0}, 1 / \rho_{0}^{\prime}\right)$ since the constants $m_{A}$ and $m_{A}^{\prime}$ are monotonically nondecreasing with $|\rho|$. Now, the system (2.1) remains stable for all

$$
\rho \in\left[-\rho_{0}^{*}, \rho_{0}^{*}\right] \subseteq\left(\left[-\rho_{0}, \rho_{0}\right] \cap\left[-\frac{1}{\rho_{0}^{\prime}}, \frac{1}{\rho_{0}^{\prime}}\right]\right) .
$$

(ii) A sufficient condition for the closed-loop system obtained from (2.1), with the same controller to remain stable irrespective of whether the number of open-loop unstable of zeros or poles of (2.1) and (2.2) is identical or not, is that the inequality

$$
\left|Q_{0}(j \omega) \frac{A^{*^{\prime}}(j \omega)}{D(j \omega)}+T_{0}(j \omega) \frac{B^{*^{\prime}}(j \omega)}{D(j \omega)}\right|<1
$$

holds for all $\omega \in \mathbb{R}_{0}^{+}$from Rouchés theorem, where $Q_{0}(s)=1-T_{0}(s)$ is the sensitivity function of the (nominal) closed-loop system obtained from (2.2) with the same controller of transfer function $c(s)$. If $T_{0}$ is stable with no critically stable poles, then $\gamma=\left\|T_{0}\right\|_{\infty}<\infty$. Also, $Q_{0}(s)=1-T_{0}(s)$. Then (A.18) holds for all $\omega \in \mathbb{R}_{0}^{+}$if

$$
\begin{aligned}
& |\rho|\left[(1-\gamma)\left|\frac{A^{*^{\prime}}(j \omega)}{D(j \omega)}\right|+\gamma\left|\frac{B^{*^{\prime}}(j \omega)}{D(j \omega)}\right|\right\rfloor \leq|\rho| \rho_{0 T}^{\prime}<1 \quad \text { if } \gamma<1, \\
& |\rho|\left[(1+\gamma)\left|\frac{A^{*^{\prime}}(j \omega)}{D(j \omega)}\right|+\gamma\left|\frac{B^{*^{\prime}}(j \omega)}{D(j \omega)}\right|\right\rfloor \leq|\rho| \rho_{0 T}^{\prime}<1 \quad \text { if } \gamma \geq 1,
\end{aligned}
$$

and the result follows by calculating the upper bounds for $\left|\Delta_{A}\left(j \omega, e^{-j \omega h}\right)\right|$ and $\mid \Delta_{B}(j \omega$, $\left.e^{-j \omega h}\right) \mid$ as $\omega$ takes values in $\mathbb{R}_{0}^{+}$in a similar way as in (i), by using the new definitions of the real constants of (3.1) and (3.3) as $m_{A T}, m_{B T}, m_{A T}^{\prime}, m_{B T}^{\prime}$, and $\rho_{0 T}^{\prime}$, from the identities (A.13d), (A.13e).

Sketch of proof of Theorem 3.2. It is similar to that of Theorem 3.1 by redefining the constants (3.1) as indicated after substituting (A.13d), (A.13e) into (A.13a), (A.13b) to prove the stability of (A.9a) by applying Rouchés theorem on zeros of analytic complex functions to its second right-hand side. Since the redefined constants replacing those in (3.1) are delay-dependent, the obtained result is dependent on the delays type.

Sketch of proof of Theorem 3.4. It is similar to that of Theorems 3.1, 3.2 by redefining the constants (3.1) as indicated and then using a comparison of the transfer function $\bar{P}_{0}(s)$ of the delay-free system (2.3) with that of (2.1) (Lemma 2.1(ii)); that is, $P(s)$, since $\mid e^{-j \omega h}-$ $1 \mid \leq 2$ for all real $\omega$ and all $h \geq 0$, which allows quoting the proofs of Theorems 3.1, 3.2 by replacing $\rho_{0}^{\prime} \rightarrow(1 / 2) \rho_{0}^{\prime}$ before guaranteeing similar conditions to (A.19) for stability. 
The (dependent on the delays) robust stability conditions of Theorem 3.2 may be weakened by using the subsequent result that applies Rouchés theorem for $N=n$ and $M=q$ and each nonnegative frequency $\omega$.

LemmA A.2 (auxiliary result for weaker robust stability conditions). Consider a quasipolynomial $p\left(s, e^{-h s}\right)=\sum_{i=0}^{N} \sum_{k=0}^{M} p_{i k} e^{-k h s} s^{i}$ of real coefficients and degrees $\partial_{s} p=N$ and $\partial_{\mu} p=$ $M$ with respect to $s$ and $\mu=e^{-h s}$, respectively. Thus, for any imaginary complex number $s=j \omega$ with $j=\sqrt{-1}$ being the imaginary unity, one gets

$$
\begin{aligned}
p\left(j \omega, e^{-j \omega h}\right)= & p_{1}(\omega)+j p_{2}(\omega)=p_{0}(j \omega)+\Delta p\left(j \omega, e^{-j \omega h}\right) \\
& \times\left(p_{10}(\omega)+j p_{20}(\omega)\right)+\left(\Delta p_{1}(\omega)+j \Delta p_{2}(\omega)\right),
\end{aligned}
$$

with $p_{0}(j \omega):=D(j \omega)=p_{10}(\omega)+j p_{20}(\omega)$, and

$$
\begin{aligned}
p_{1}(\omega) & =\sum_{i=0}^{N / 2-1} \sum_{k=0}^{M}(-1)^{i}\left(p_{2 i, k} \cos k \omega h+\omega p_{2 i+1, k} \sin k \omega h\right) \omega^{2 i}+\sum_{k=0}^{M} p_{N k} \omega^{N} \cos k \omega h, \\
p_{2}(\omega) & =\sum_{i=0}^{N / 2-1} \sum_{k=0}^{M}(-1)^{i}\left(\omega p_{2 i+1, k} \cos k \omega h-p_{2 i, k} \sin k \omega h\right) \omega^{2 i}-\sum_{k=0}^{M} p_{N k} \omega^{N} \sin k \omega h
\end{aligned}
$$

if $N$ is even, and

$$
\begin{aligned}
& p_{1}(\omega)=\sum_{i=0}^{(N-1) / 2} \sum_{k=0}^{M}(-1)^{i}\left(p_{2 i, k} \cos k \omega h+\omega p_{2 i+1, k} \sin k \omega h\right) \omega^{2 i}, \\
& p_{2}(\omega)=\sum_{i=0}^{(N-1) / 2} \sum_{k=0}^{M}(-1)^{i}\left(\omega p_{2 i+1, k} \cos k \omega h-p_{2 i, k} \sin k \omega h\right) \omega^{2 i}
\end{aligned}
$$

if $N$ is odd and $p_{0}(\omega)=p_{10}(\omega)+j p_{10}(\omega)$, where

$$
\begin{aligned}
& p_{10}(\omega)=\sum_{i=0}^{N}(-1)^{i}\left(p_{k 0} \cos k \omega h+\omega p_{k 1} \sin k \omega h\right), \\
& p_{20}(\omega)=\sum_{k=0}^{N}(-1)^{i}\left(\omega p_{k 1} \cos k \omega h-p_{k 0} \sin k \omega h\right) .
\end{aligned}
$$

As a result, $p\left(s, e^{-h s}\right)$ is stable (or, so called, strictly Hurwitzian; s, i.e., $p\left(s, e^{-h s}\right) \neq 0$ ) for $\operatorname{Re} s \leq 0$ if $p_{0}(s)=\sum_{i=0}^{n} p_{i 0} s^{i}$ is a stable polynomial (i.e., $\sum_{i=0}^{n} p_{i 0} s^{i} \neq 0$ for $\operatorname{Re} s \leq 0$ ) and, furthermore,

$$
\Delta p_{1}^{2}(\omega)+\Delta p_{2}^{2}(\omega)<p_{10}^{2}(\omega)+p_{20}^{2}(\omega)
$$

for all $\omega \in \mathbb{R}_{0}^{+}$. Assume that $\Delta p_{i}(\omega)=\rho \Delta p_{i}^{\prime}(\omega)=\rho\left(\Delta p_{i}^{\prime \prime}(\omega)+\rho \Delta \bar{p}_{i}^{\prime \prime}(\omega)\right)$ for $i=1,2$ and all $\omega \in \mathbb{R}_{0}^{+}$. Thus, since $\Delta p_{1}^{2}(\omega)+\Delta p_{2}^{2}(\omega)>0$ for all $\omega \in \mathbb{R}_{0}^{+}$, it always exists from (A.24) a sufficiently small $\rho_{0}^{*}>0$ such that $p\left(s, e^{-h s}\right)$ is stable for all $|\rho| \leq \rho_{0}^{*}$. 
Proof. The decomposition into real and imaginary parts of the quasipolynomial becomes direct from the identity

$$
p\left(j \omega, e^{-j \omega h}\right)=\sum_{i=0}^{N} \sum_{k=0}^{M}(j)^{i} p_{i k} \omega^{i}(\cos k \omega h-j \sin k \omega h)
$$

since $\cos (-\varphi)=\cos \varphi$ and $\sin (-\varphi)=-\sin \varphi$; and if $i \in \mathbb{Z}^{+}$is any positive integer fulfilling $i=\dot{4}$ then $j^{i}=1$ and $j^{i+1}=j$; and if $\dot{2}=i \neq \dot{4}$ then $j^{i}=-1$ and $j^{i+1}=-j$. The robust stability condition follows from Rouchés theorem for zeros of the complex functions $p_{0}(j \omega)$ and $p\left(j \omega, e^{-j \omega h}\right)=p_{0}(j \omega)+\Delta p\left(j \omega, e^{-j \omega h}\right)$ from the imaginary complex axis to $C$.

The proof follows directly from Theorem 3.1 and Lemma A.2.

\section{Acknowledgments}

The author is very grateful to the Spanish Ministry of Science and Technology and to UPV/EHU for their partial support of this work through Research Grants DPI 200300164 and 9/UPV/EHU 00I06.00I06-15263/2003 (Research Groups).

\section{References}

[1] H. Bourlès, $\alpha$-stability of systems governed by a functional-differential equation-extension of results concerning linear delay systems, International Journal of Control 45 (1987), no. 6, 22332234.

[2] S. D. Brierley, J. N. Chiasson, E. B. Lee, and S. H. Żak, On stability independent of delay for linear systems, IEEE Transactions on Automatic Control 27 (1982), no. 1, 252-254.

[3] J. Chen, On computing the maximal delay intervals for stability of linear delay systems, IEEE Transactions on Automatic Control 40 (1995), no. 6, 1087-1093.

[4] C. Corduneanu and N. Luca, The stability of some feedback systems with delay, Journal of Mathematical Analysis and Applications 51 (1975), no. 2, 377-393.

[5] R. F. Datko, Remarks concerning the asymptotic stability and stabilization of linear delay differential equations, Journal of Mathematical Analysis and Applications 111 (1985), no. 2, 571-584.

[6] _ Time-delayed perturbations and robust stability, Differential Equations, Dynamical Systems, and Control Science (K. D. Elworthy, W. N. Everitt, and E. B. Lee, eds.), Lecture Notes in Pure and Applied Mathematics Series, vol. 152, Marcel Dekker, New York, 1994, pp. 457-468.

[7] M. De La Sen, On some structures of stabilizing control laws for linear and time-invariant systems with bounded point delays and unmeasurable states, International Journal of Control 59 (1994), no. 2, 529-541.

[8] _ Allocation of poles of delayed systems related to those associated with their undelayed counterparts, Electronics Letters 36 (2000), no. 4, 373-374.

[9] _ Preserving positive realness through discretization, Positivity 6 (2002), no. 1, 31-45.

[10] P. Dorato, L. Fortuna, and G. Muscato, Robust Control for Unstructured Perturbations-An Introduction, Lecture Notes in Control and Information Sciences, vol. 168, Springer, Berlin, 1992, series editors M. Thoma and A. Wyner.

[11] G. F. Franklin and J. D. Powell, Digital Control of Dynamic Systems, Addison-Wesley, Massachusetts, 1980.

[12] K. Gu, Discretized LMI set in the stability problem of linear uncertain time-delay systems, International Journal of Control 68 (1997), no. 4, 923-934.

[13] J. K. Hale, E. F. Infante, and F. S. P. Tsen, Stability in linear delay equations, Journal of Mathematical Analysis and Applications 105 (1985), no. 2, 533-555. 
[14] T. Kailath, Linear Systems, Prentice-Hall Information and System Sciences Series, Prentice-Hall, New Jersey, 1980.

[15] E. W. Kamen, On the relationship between zero criteria for two-variable polynomials and asymptotic stability of delay differential equations, IEEE Transactions on Automatic Control 25 (1980), no. 5, 983-984.

[16] D. Kincaid and W. Cheney, Numerical Analysis. Mathematics of Scientific Computing, Brooks/Cole, California, 1991.

[17] H. Kwakernaak, Uncertainty Models and the Design of Robust Control Systems, edited by J. Ackerman, Lecture Notes in Control and Information Sciences Series, no. 70, Springer, Berlin, 1992, series editor M. Thoma.

[18] J. S. Luo and P. P. J. van den Bosch, Independent of delay stability criteria for uncertain linear state space models, Automatica 33 (1997), no. 2, 171-179.

[19] J. M. Ortega, Numerical Analysis. A Second Course, Computer Science and Applied Mathematics, Academic Press, New York, 1972.

[20] A. D. Wunsch, Complex Variables with Applications, 2nd ed., Addison-Wesley, Massachusetts, 1994.

[21] B. Xu, Stability robustness bounds for linear systems with multiple time-varying delayed perturbations, International Journal of Systems Science 28 (1997), no. 12, 1311-1317.

[22] Stability criteria for linear time-invariant systems with multiple delays, Journal of Mathematical Analysis and Applications 252 (2000), no. 1, 484-494.

M. De La Sen: Departamento de Electricidad y Electrónica, Instituto de Investigación y Desarrollo de Procesos (IIDP), Facultad de Ciencias, Universidad del País Vasco, Leioa (Bizkaia), Aptdo, 644 de Bilbao, 48080-Bilbao, Spain E-mail address: wepdepam@lg.ehu.es 


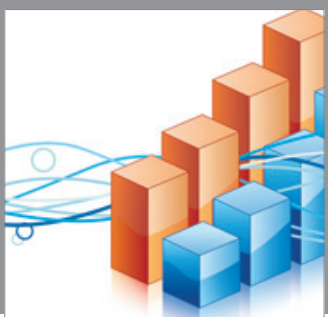

Advances in

Operations Research

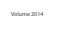

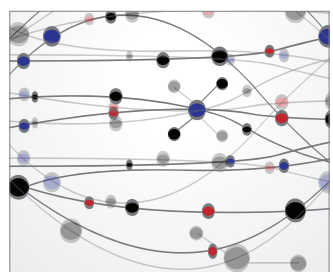

\section{The Scientific} World Journal
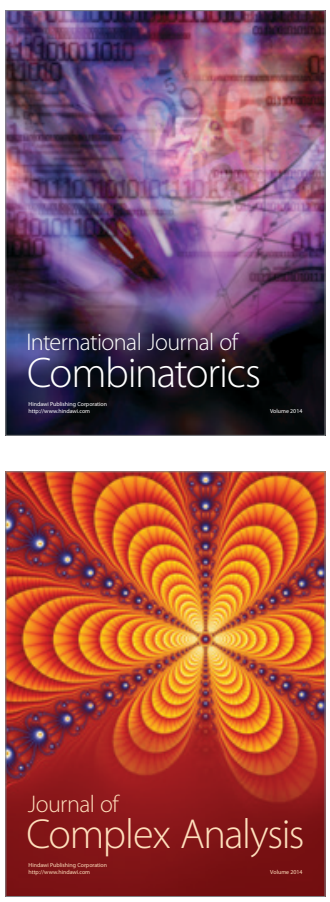

International Journal of

Mathematics and

Mathematical

Sciences
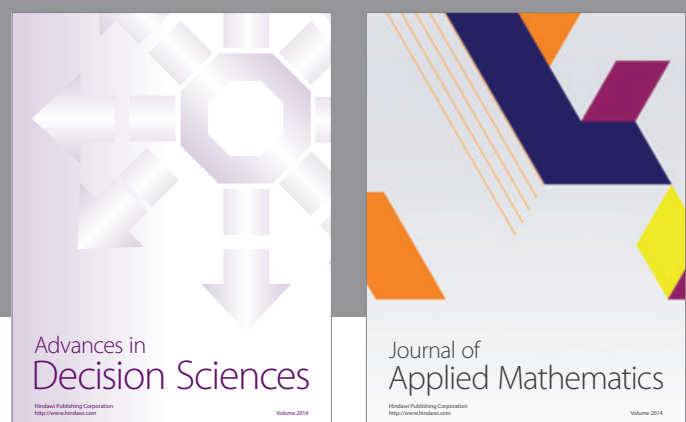

Journal of

Applied Mathematics
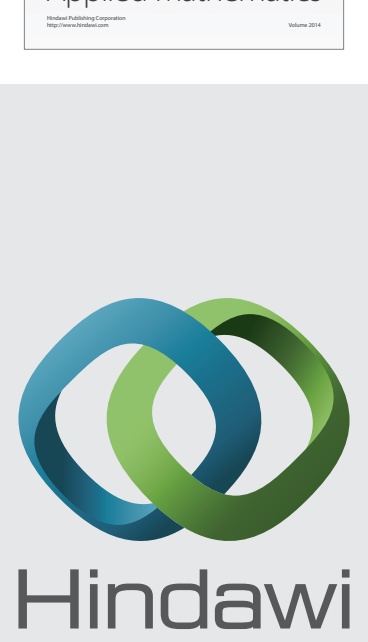

Submit your manuscripts at http://www.hindawi.com
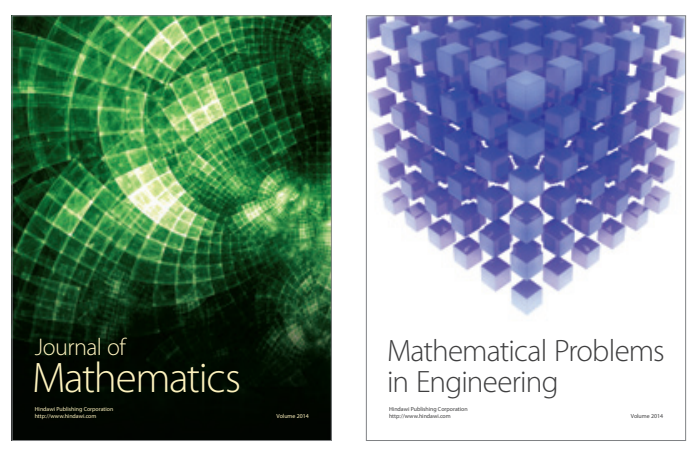

Mathematical Problems in Engineering
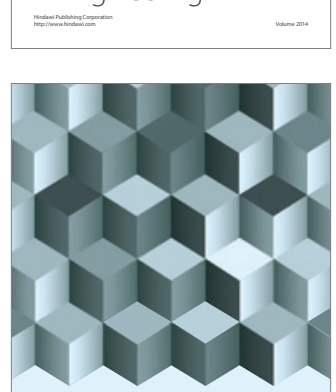

Journal of

Function Spaces
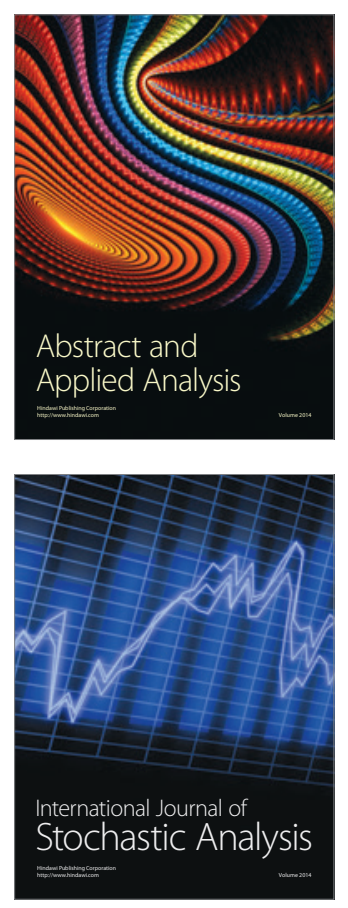

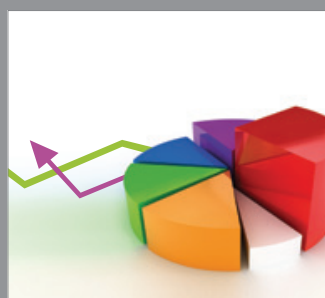

ournal of

Probability and Statistics

Promensencen
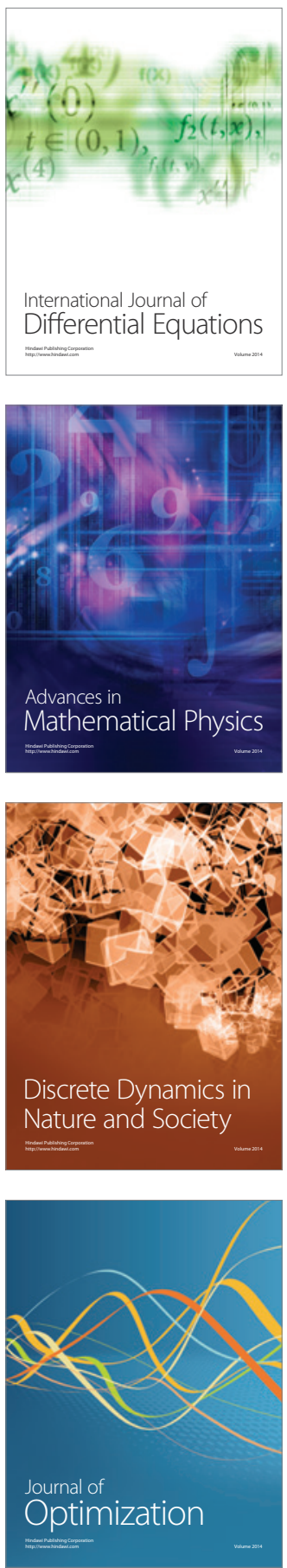